\title{
Coronary artery calcium in patients with schizophrenia
}

Trine Trab ${ }^{1,2,3^{*}}$, Rubina Attar ${ }^{2}$, Svend Eggert Jensen ${ }^{2,3}$, Simon Grøntved', Jens Brøndum Frøkjær ${ }^{3,4}$, Christoffer Polcwiartek ${ }^{2}$ and René Ernst Nielsen ${ }^{1,3}$

\begin{abstract}
Background: Coronary heart disease (CHD) is a major cause of increased mortality rates in patients with schizophrenia. Moreover, coronary artery calcium (CAC) score is associated with CHD. We hypothesized that patients with schizophrenia have more CAC than the general population and aimed to investigate the CAC score in patients with schizophrenia compared to norms based on the general population. Additionally, this study investigated if age, sex, diabetes, dyslipidemia and smoking were associated with the CAC score.

Methods: In a cross-sectional study, 163 patients with schizophrenia underwent cardiac computed tomography, and the CAC score was measured and compared to norms by classifying the CAC scores in relation to the age- and gender matched norm 50th, 75th and 90th percentiles. Logistic and linear regression were carried out to investigate explanatory variables for the presence and extent of CAC, respectively.

Results: A total of $127(77.9 \%)$ patients had a CAC score below or equal to the matched 50th, $20(12.3 \%)$ above the 75th and nine (5.5\%) above the 90th percentile. Male sex $(P<0.05)$, age $(P<0.001)$ and smoking $(P<0.05)$ were associated with the presence of CAC while age $(P<0.001)$ and diabetes $(P<0.01)$ were associated with the extent of CAC.

Conclusions: The amount of CAC in patients with schizophrenia follows norm percentiles, and variables associated with the CAC score are similar in patients with schizophrenia and the general population. These findings indicate that the CAC score may not be sufficient to detect the risk of CHD in patients with schizophrenia. Future studies should explore other measures of subclinical CHD, including measures of peripheral atherosclerosis or cardiac autonomic neuropathy to improve early detection and intervention.
\end{abstract}

Trial registration: ClinicalTrials.gov Identifier: NCT02885792, September 1, 2016.

Keywords: Coronary artery disease, Schizophrenia, Vascular Calcification

\section{Background}

Compared to the general population, patients with schizophrenia have a shorter life expectancy and experience relatively more deaths from unnatural causes, e.g. suicide $[1,2]$. However, most life years lost are attributable to physical disease including coronary

\footnotetext{
*Correspondence: t.trab@rn.dk

${ }^{1}$ Department of Psychiatry, Aalborg University Hospital, Aalborg, Denmark 2Department of Cardiology, Aalborg University Hospital, Aalborg, Denmark Full list of author information is available at the end of the article
}

heart disease (CHD) [1-3]. Patients with schizophrenia have an increased prevalence of CHD, cardiovascular mortality and cardiovascular risk factors consisting of diabetes, dyslipidemia, hypertension and central obesity compared to the general population [4-7]. This risk profile is probably due to higher rates of smoking, unhealthy lifestyle, physical inactivity, side effects to antipsychotic medicine $[4,6]$ and reduced compliance [8]. In European countries, cardiovascular mortality rates have declined over the last decades

(c) The Author(s). 2021 Open Access This article is licensed under a Creative Commons Attribution 4.0 International License, which permits use, sharing, adaptation, distribution and reproduction in any medium or format, as long as you give appropriate credit to the original author(s) and the source, provide a link to the Creative Commons licence, and indicate if changes were made. The images or other third party material in this article are included in the article's Creative Commons licence, unless indicated otherwise in a credit line to the material. If material is not included in the article's Creative Commons licence and your intended use is not permitted by statutory regulation or exceeds the permitted use, you will need to obtain permission directly from the copyright holder. To view a copy of this licence, visit http://creativecommons.org/licenses/by/4.0/ The Creative Commons Public Domain Dedication waiver (http://creativecommons.org/publicdomain/zero/1.0/) applies to the data made available in this article, unless otherwise stated in a credit line to the data. 
due to improved diagnostic methods, treatment options and lifestyle changes [9]. However, patients with schizophrenia receive less pharmacological treatment of cardiovascular risk factors and lower prescription rates of cardioprotective drugs following myocardial infarction compared to the general population [10, 11]. Late detection of risk factors [4] and higher prevalence of unrecognized myocardial infarction have been reported [12], which might be due to atypical presentation and lack of symptom recognition [13]. Thus, tools for early detection of CHD in patients with schizophrenia are needed to establish preventive strategies and reduce the risk of cardiovascular mortality. The amount of coronary artery calcium (CAC) has shown to be associated with CHD mortality independent of other cardiovascular risk factors in the general population [14-16]. The presence and extent of coronary artery calcium can be quantified by the use of noninvasive cardiac computed tomography (CCT) using the CAC score, which correlates with the actual amount of histologically detectable calcium in the coronary arteries detected post-mortem [17]. The CAC score has shown to improve cardiovascular risk assessment when added to traditional risk factors in the general population $[15,16,18]$. Established risk models for prediction of CHD may not be sufficient in the determination of cardiovascular risk in patients with schizophrenia [19], and measurement of CAC scores could possibly add prognostic information in the same way as in the general population. One other study showed no difference in CAC scores in a group of patients diagnosed with schizophrenia or bipolar disorder referred for CCT on clinical indication compared to other people with a registered CCT [20]. Traditional risk factors such as diabetes, dyslipidemia, smoking, sex and age have shown to be associated with the CAC scores in the general population [20,21].

However, the potential role of CAC score measurement for early recognition of subclinical CHD has not yet been investigated in patients with schizophrenia screened without a clinical indication for conducting the diagnostic procedure.

In this study, we hypothesized that patients with schizophrenia have a higher coronary artery calcium score compared to the general population. Thus, the aim of this study was to investigate the coronary artery calcium score in patients with schizophrenia, who were not referred on clinical indication as compared to reference norms based on the general population. Moreover, we aimed to investigate if age, sex, diabetes, dyslipidemia and smoking were associated with the coronary artery calcium score.

\section{Methods}

\section{Study design and subjects}

In a clinical cross-sectional study, a total of 163 patients diagnosed with schizophrenia underwent CCT scan and measurement of the CAC score, which represents the total amount of CAC summed for the coronary arteries using densities and areas of calcified lesions. Based on the CAC score, the risk of future CHD is categorized into four categories: $0=$ very low risk, $1-99=$ mildly increased risk, 100-299 = moderately increased risk, 300$1000=$ moderate to severely increased risk [22]. In addition, the score is commonly reported in relation to age- and gender matched percentiles, and values above the 75th percentile are of clinical interest in connection with increased risk of CHD [23-25].

First, the population was categorized into two groups $(\mathrm{CAC}$ score $=0$ and $\mathrm{CAC}$ score $>0)$ representing the $\mathrm{ab}$ sence or presence of any CAC given the increased survival rates in patients with a CAC score of zero [26] and the general zero inflated distribution of the CAC score [27]. Second, the population was categorized in relation to the age- and gender matched CAC score norm percentiles used in Danish clinical practice [25].

Patients were included from outpatient clinics in the North Denmark Region and inclusion criteria were age $\geq 18$ years, residency in the North Denmark Region and diagnosed with F20 (schizophrenia) or F25 (schizoaffective disorder) according to the International Classification of Diseases, Tenth Revision (ICD-10). Exclusion criteria were pregnancy, lactation, claustrophobia or inability to cooperate on the planned examinations. All participants provided written informed consent.

Data was collected at Aalborg University Hospital, Denmark between December 2015 and October 2019 as part of a clinical prospective cohort study conducted by the CardioSchizoStudyGroup [28].

\section{Assessment of coronary artery calcium}

The CAC score was assessed from CCT scans with a 128-slice CT scanner (Siemens Healthcare GmbH, Germany). The examination was performed in supine position (tube voltage $120 \mathrm{kV}$, tube current $50 \mathrm{mAs}$, tube rotation time of $0.28 \mathrm{~s}$ ) with a slice thickness of $3 \mathrm{~mm}$ and without intravenous contrast administration. Two observers (medical student TT and senior cardiologist SEJ) independently marked all calcified lesions with relation to the coronary arteries distinguishable from the aorta. The imaging analysis software SIEMENS Workstation syngo.via was used to calculate the CAC score as a sum of scores for all marked lesions with a minimum attenuation of 130 Hounsfield Units and a minimum area of two contiguous voxels according to the Agatston method [29]. This method is a well-established standardized CAC scoring method and is considered the gold standard for quantification of CAC [23, 30]. In the case of disagreement between the observers, images were reanalyzed to reach consensus. 


\section{Baseline characteristics}

The severity of schizophrenia was assessed by an experienced psychiatric nurse using the overall severity from the standardized Clinical Global ImpressionSchizophrenia rating scale (CGI-SCH) [31]. The severity was given a rating of one to seven points categorizing the patients as either normal, not at all ill (one point), borderline mentally ill (two points), mildly ill (three points), moderately ill (four points), markedly ill (five points), severely ill (six points) or among the most extremely ill patients (seven points). Clozapine treatment indicating resistance to treatment was defined as present if current use of clozapine was reported. The duration of schizophrenia was defined as years since the patients were diagnosed with schizophrenia assessed by the first appearance of the diagnosis in the medical records. Blood samples were collected to determine serum concentration of glycated hemoglobin (HbA1c), total cholesterol, triglyceride, high-density lipoprotein (HDL) and low-density lipoprotein (LDL). Smoking was defined as present if the participant reported any current or previous smoking. Diabetes was defined as present if HbA1c $\geq 48 \mathrm{mmol} / \mathrm{l}$, or if current antidiabetic medication was reported. Blood pressure was measured on each arm after ten minutes of rest and mean systolic blood pressure was calculated. Dyslipidemia was defined as present if current lipid lowering medication was reported, or if total cholesterol, triglyceride, HDL or LDL exceeded specific thresholds. The thresholds were determined from the estimated ten-year risk for fatal cardiovascular events and whether diabetes was present. The absolute risk was estimated in patients $\geq 40$ years and the relative risk in patients $<40$ years in accordance with the SCORE algorithm for low risk countries [32]. The estimations were based on age, total cholesterol, mean systolic blood pressure and smoking status. Hypertension was defined as present if mean systolic blood pressure was $\geq 140$ $\mathrm{mmHg}$, mean diastolic blood pressure was $\geq 90 \mathrm{mmHg}$ or current antihypertensive medication was reported. Obesity was defined as present if body mass index (BMI) was $\geq 30$ determined by height and weight.

\section{Statistical analysis}

Baseline characteristics were analyzed according to type, calculating percentages and frequencies for categorical data and means and standard deviations (SD) for continuous data and for CGI-SCH points. The CAC score was reported as percentiles. Baseline characteristics in patients with a CAC score of zero were compared to baseline characteristics in patients with a CAC score above zero using chi-squared tests for categorical data and t-test for continuous data. The CAC scores were categorized into $0,1-99,100-299$ and $\geq 300$ and compared to age- and gender matched norm percentiles used in clinical practice in Denmark [25] graphically and by reporting the percentage of CAC scores below or equal to the 50th percentile, above the 75 th percentile and above the 90th percentile. Previous studies found associations between age, male sex, diabetes, dyslipidemia, smoking and hypertension as explanatory variables and CAC score as outcome in the general population [20, 21]. We aimed to explore the association between these variables and the CAC score in patients with schizophrenia by regression analysis. The number of patients in the present study, especially the number of patients having a CAC score above zero $(n=49)$, led us to prioritize and limit the number of covariates included in the main analysis. Even though hypertension is relevant for the analysis, the forementioned prioritization led us to not include blood pressure. This was done, as we do believe that blood pressure is most likely to be influenced by external factors such as measurement in a clinical setting and most likely to fluctuate over time and. Thus, blood pressure measured in a clinical setting might not be good enough proxy for hypertension to warrant adding the extra covariate in the regression analysis. The CAC score was not normally distributed; however, CAC scores above zero were normally distributed after logtransformation determined using histogram, QQ-plot and Shapiro Wilk test for normality. Therefore, the regression analysis was performed in two steps, a method also applied in previous studies [21,33]. First, a logistic regression model was utilized to assess the correlation between age, sex, diabetes, dyslipidemia and smoking as explanatory variables and a CAC score above zero as outcome. Second, a linear regression model was applied on log-transformed CAC scores above zero to assess the correlation between age, sex, diabetes, dyslipidemia and smoking as explanatory variables and the extent of coronary artery calcium as outcome measure. Both steps consisted of five univariate models with age, sex, diabetes, dyslipidemia and smoking respectively as explanatory variables and a CAC score above zero as outcome and one multivariate model with age, sex, diabetes, dyslipidemia and smoking as explanatory variables and CAC score as outcome. Results from the logistic regression were reported as odds ratios (OR) and coefficients from the linear regression were transformed to percentage change in CAC score to ease interpretation. A sensitivity analysis on treatment with lipid lowering medication, antidiabetic medication, serum concentration of HbA1c, triglyceride, HDL and LDL was carried out. Furthermore, a second posthoc sensitivity analysis was carried out including logistic and linear regression with hypertension, obesity, age, sex and smoking as explanatory variables and CAC score as outcome. Obesity was included in this analysis since one previous study [20] has shown association between BMI and the CAC 
score in patients with schizophrenia. Hypertension was included since previous studies have shown association with the CAC score in patients with schizophrenia and the general population [20, 34, 35] A $P$-value $<0.05$ was considered statistically significant. All analyses were performed using STATA version 16 (StataCorp LP, College Station, Texas, USA).

\section{Results}

This study included 163 patients (96 (58.9\%) males). The mean age was $48.2(\mathrm{SD} \pm 10.4)$ years, ranging from 24 to 75 years. The mean duration of schizophrenia was 19.7 $(\mathrm{SD} \pm 8.2$ ) years, ranging from 3 to 46 years. According to the CGI-SCH rating scale, one patient $(0.6 \%)$ was categorized as normal, not at all ill, $23(15 \%)$ as borderline mentally ill, $45(29 \%)$ as mildly ill, $49(32 \%)$ as moderately ill, $26(17 \%)$ as markedly ill, $10(6 \%)$ as severely ill and one $(0.6 \%)$ as among the most extremely ill. A total of $41(25.2 \%)$ patients received clozapine treatment. Further baseline characteristics are given in Table 1, and baseline characteristics stratified by group (CAC score $=$ 0 and CAC score > 0) are given in Table 2. Less than 5\% missing data was found on $\mathrm{HbA1c}$, total cholesterol, triglyceride, HDL, LDL, smoking and lipid lowering medication due to withdrawal of consent, unwillingness or inability to participate in these examinations. This was considered missing at random, and imputation (mean for continuous variables and random for categorical variables) was carried out to deal with the missing data for regression modelling.

The CAC score ranged from zero to 3448 with a mean of 86.5 ( $\mathrm{SD} \pm 376$ ), 25th percentile of zero, 50th percentile of zero, 75th percentile of six and 90th percentile of 114. A total of $114(69.9 \%)$ patients had a CAC score of zero including 65 (39.9\%) males and 49 (30.1\%) females, $31(19.0 \%)$ had a CAC score in the range 1-99, seven (4.3\%) in the range $100-299$ and $11(6.8 \%) \geq 300$. A total of $127(77.9 \%)$ patients including $76(46.6 \%)$ males and 51 (31.3\%) females had a CAC score below or equal to the age- and gender matched norm 50th percentile. All patients with a CAC score exactly equal to the age- and gender matched norm 50th percentile had a CAC score of zero. A total of $20(12.3 \%)$ patients including seven (7.3\%) males and 13 (19.4\%) females had a CAC score above the age- and gender matched norm 75 th percentile and one male patient $(0.6 \%)$ had a CAC above zero and exactly equal to the 75 th percentile. A total of nine (5.5\%) patients including three (3.1\%) males and six (9.0\%) females had a CAC score above the norm 90th percentile. Fig. 1 illustrates the CAC scores in relation to age- and gender matched norm percentiles. Patients with a CAC score above zero were older $(P<0.001)$, had an increased duration of schizophrenia $(P<0.001)$ and
Table 1 Baseline characteristics

\begin{tabular}{|c|c|}
\hline & Total \\
\hline N (\%) & $163(100)$ \\
\hline Age, mean (SD) & $48.2(10.4)$ \\
\hline Male sex, n (\%) & $96(58.9)$ \\
\hline Dyslipidemia, n (\%) & 109 (66.9) \\
\hline Diabetes, n (\%) & $34(20.9)$ \\
\hline Smoking, n (\%) & $124(76.1)$ \\
\hline Hypertension, n (\%) & $62(38.0)$ \\
\hline Obesity, n (\%) & $63(38.7)$ \\
\hline LDL, unmeasurable, n (\%) & $12(7.4)$ \\
\hline mean (SD) & $2.5(0.9)$ \\
\hline $\mathrm{HDL}$, mean (SD) & $1.3(0.4)$ \\
\hline Triglyceride, mean (SD) & $2.3(2.3)$ \\
\hline \multicolumn{2}{|l|}{ CAC score } \\
\hline 25th percentile & 0 \\
\hline 50th percentile & 0 \\
\hline 75th percentile & 6 \\
\hline 90th percentile & 114 \\
\hline \multicolumn{2}{|l|}{ Duration of schizophrenia } \\
\hline n (\%) & $141(100)$ \\
\hline mean years (SD) & $19.7(8.2)$ \\
\hline \multicolumn{2}{|l|}{ Severity of schizophrenia ${ }^{a}$} \\
\hline n (\%) & $155(100)$ \\
\hline Normal, not at all ill (1 point) & $1(0.6)$ \\
\hline Borderline mentally ill (2 points) & $23(15)$ \\
\hline Mildly ill (3 points) & $45(29)$ \\
\hline Moderately ill (4 points) & $49(32)$ \\
\hline Markedly ill (5 points) & $26(17)$ \\
\hline Severely ill (6 points) & $10(6)$ \\
\hline Among the most extremely ill (7 points) & $1(0.6)$ \\
\hline CGI-SCH score ${ }^{a}$, mean (SD) & $3.7(1.2)$ \\
\hline Clozapine treatment, n (\%) & $41(25.2)$ \\
\hline
\end{tabular}

${ }^{a}$ Based on the $\mathrm{CGI}-\mathrm{SCH}$ rating scale for overall severity.

higher rates of dyslipidemia $(P<0.05)$ and smoking $(P<$ $0.01)$ than patients with a CAC score of zero.

\section{Regression analysis}

In the univariate logistic regression models, age (OR 1.19; 95\% CI 1.13-1.20; $P<0.001$ ), dyslipidemia (OR 2.23; 95\% CI 1.03-5.02; $P<0.05$ ) and smoking (OR 3.74, 95\% CI 1.36-10.3; $P<0.01$ ) were significantly associated with a CAC score above zero. However, in the multivariate model, the association between dyslipidemia and a CAC score above zero was no longer significant (OR 1.84; $95 \%$ CI $0.66-5.14 ; P>0.05)$. In the multivariate model, the association between age and a CAC score above zero remained significant (OR 1.20; 95\% CI 1.13- 
Table 2 Baseline characteristics comparison between groups CAC score $=0$ and CAC score $>0$

\begin{tabular}{|c|c|c|c|}
\hline Variables & CAC score $=0$ & CAC score $>0$ & $P$-value \\
\hline n (\%) & $114(69.9)$ & $49(30.1)$ & \\
\hline Age, mean (SD) & $44.5(9.1)$ & $56.9(7.7)$ & $<0.001$ \\
\hline Male sex, n (\%) & $65(57.0)$ & $31(63.3)$ & 0.457 \\
\hline Dyslipidemia, n (\%) & $70(63.6)$ & 39 (79.6) & 0.045 \\
\hline Diabetes, n (\%) & $20(17.5)$ & $14(28.6)$ & 0.112 \\
\hline Smoking, n (\%) & $80(70.2)$ & $44(89.8)$ & 0.007 \\
\hline Hypertension, n (\%) & $38(33.3)$ & $24(48.8)$ & 0.059 \\
\hline Obesity, n (\%) & $46(40.4)$ & $17(34.7)$ & 0.496 \\
\hline LDL, unmeasurable, n (\%) & $9(8.4)$ & $3(6.1)$ & \\
\hline LDL, measurable, n (\%) & 98 (91.6) & $46(93.9)$ & \\
\hline mean (SD) & $2.6(0.9)$ & $2.5(0.9)$ & \\
\hline $\mathrm{HDL}$, mean (SD) & $1.3(0.4)$ & $1.4(0.5)$ & \\
\hline Triglyceride, mean (SD) & $2.2(1.9)$ & $2.5(3.0)$ & \\
\hline \multicolumn{4}{|l|}{ CAC score } \\
\hline 25th percentile & & 13 & \\
\hline 50th percentile & & 35 & \\
\hline 75th percentile & & 221 & \\
\hline 90th percentile & & 783 & \\
\hline \multicolumn{4}{|l|}{ Severity of schizophrenia ${ }^{a}$} \\
\hline CGI-SCH score ${ }^{a}$, mean (SD) & $3.7(1.1)$ & $3.6(1.3)$ & 0.545 \\
\hline \multicolumn{4}{|l|}{ Duration of schizophrenia } \\
\hline mean years (SD) & $17.8(7.6)$ & $24.1(8.1)$ & $<0.001$ \\
\hline Clozapine treatment, n (\%) & $33(28.9)$ & $8(16.3)$ & 0.089 \\
\hline
\end{tabular}

Based on the CGI-SCH rating scale for overall severity.

1.20; $P<0.001)$, similar to the association between smoking and a CAC score above zero (OR 3.59; 95\% CI 1.07-12.07; $P<0.05)$. In the model, male sex was also significantly associated with a CAC score above zero (OR 2.73; 95\% CI 1.05-7.13; $P<0.05$ ). An overview of the logistic regression analysis is given in Table 3 .

The univariate linear regression of log-transformed CAC scores above zero $(n=49)$ showed that age and diabetes were significantly associated with the extent of coronary artery calcium measured as CAC score. In this model, a one-year increase in age was associated with a $15 \%$ increase in CAC score (95\% CI 7-24\%; $P<0.001$ ), while diabetes was associated with a $577 \%$ increase in CAC score (95\% CI 57-2020\%; $P<0.01$ ). Similar results were found in the linear regression multivariate model in which a one-year increase in age was associated with a $16 \%$ increase in CAC score, an association which remained statistically significant (95\% CI 9-25\%; $P<$ $0.001)$. In the multivariate model, diabetes was associated with a $419 \%$ increase in CAC score which remained statistically significant (95\% CI 68-1500\%; $P<0.01$ ). An overview of results from the linear regression models is given in Table 4. Sensitivity analyses (Table 5) showed no association between CAC score and LDL, triglyceride, $\mathrm{HDL}$, statin treatment or $\mathrm{HbA} 1 \mathrm{c}$ in multivariate logistic and linear regression. Antidiabetic medication was significantly associated with log-transformed CAC scores $(P<0.05)$, which is consistent with the association between diabetes and the extent of CAC found in the linear regression. The posthoc sensitivity analysis showed that hypertension and obesity were not associated with the CAC score (data not shown).

\section{Discussion}

In this study, the amount of coronary artery calcium in patients with schizophrenia follows age- and gender matched norms based on the general population with $77.9 \%$ below or equal to the norm 50 th percentile, $12.3 \%$ above the norm 75 th percentile and $5.5 \%$ above the norm 90th percentile. Variables independently associated with the presence of coronary artery calcium were male sex, age and smoking, and variables independently associated with the extent of coronary artery calcium were age and diabetes.

Similarly, previous studies found age, sex, smoking and diabetes to be associated with CAC score in the general 

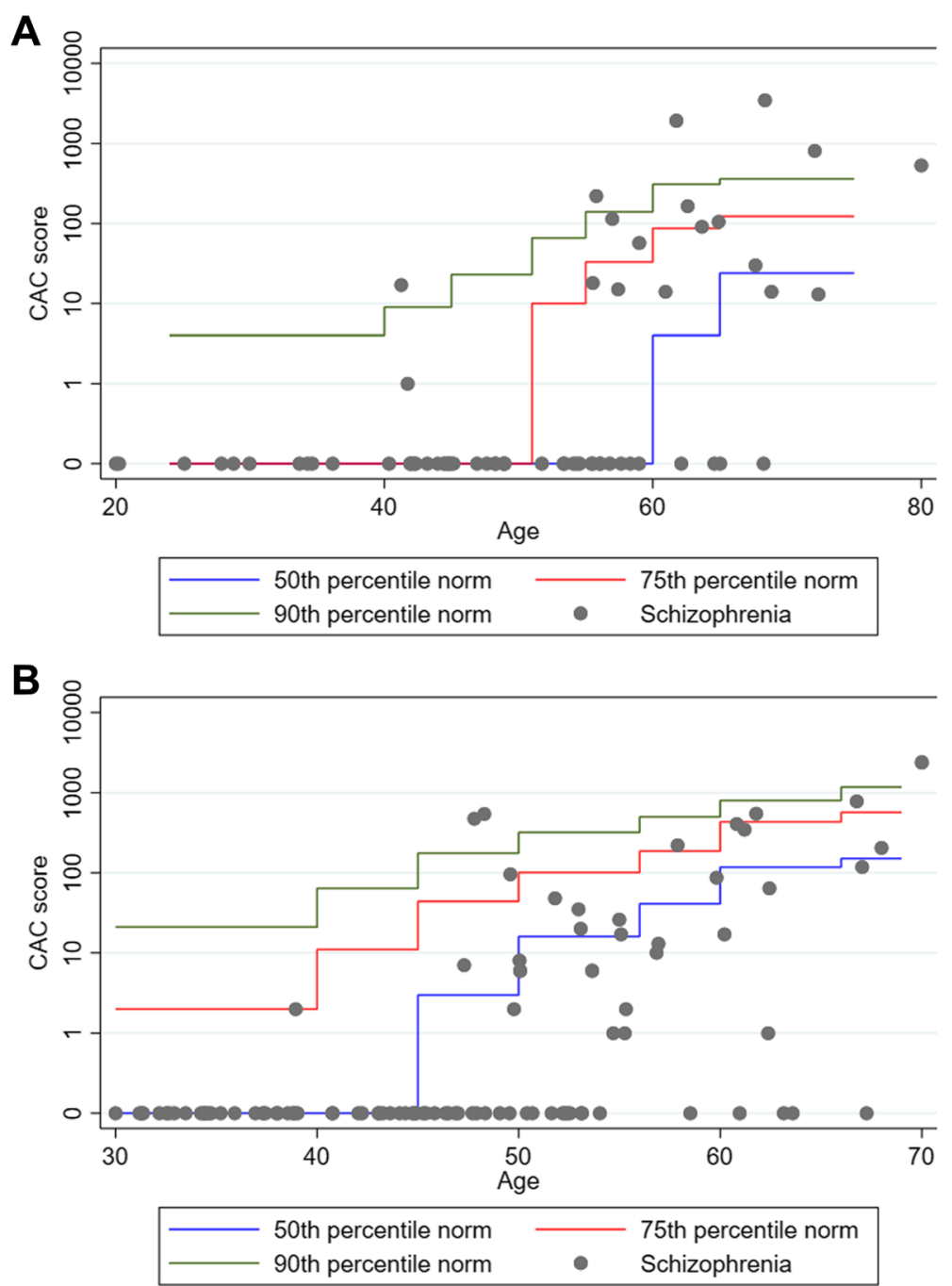

Fig. 1 The CAC scores in patients with schizophrenia represented on a logarithmic scale in relation to age-specific norm 50th, 75th and 90th percentiles divided by sex. Zero was added on the otherwise logarithmic $y$-axis to show the large amount of patients having a CAC score of zero. A Females B Males

population [20, 21, 36]. In a study by Kugathasan et al. [20] age, smoking, BMI and hypertension were found to be associated with the CAC score in a group of patients with schizophrenia or bipolar disorder all undergoing cardiac procedures on a clinical indication. However,

Table 3 Logistic regression on the presence of CAC measured as CAC score

\begin{tabular}{|c|c|c|c|c|}
\hline \multirow[t]{2}{*}{ Variables } & \multicolumn{2}{|l|}{ Univariate } & \multicolumn{2}{|l|}{ Multivariate } \\
\hline & OR (95\% CI) & $\overline{P \text {-value }}$ & OR $(95 \% \mathrm{Cl})$ & $P$-value \\
\hline Age & $1.19(1.12-1.26)$ & $<0.001$ & $1.21(1.13-1.29)$ & $<0.001$ \\
\hline Male & 1.33 & 5 & 3.15 & 0.0 \\
\hline Dyslipidemia & $2.23(1.01-4.94)$ & 0.048 & $1.48(0.52-4.23)$ & 0.465 \\
\hline Diabet & $2.19(0.98-4.90)$ & 0.057 & $1.75(0.60-5.16)$ & 0.308 \\
\hline Smoking & $3.94(1.43-10.8)$ & 0.008 & $3.95(1.16-13.48)$ & 0.028 \\
\hline
\end{tabular}

hypertension and BMI applied in posthoc sensitivity analysis in the present study showed no association with the CAC score. One possible explanation for the contradictory results could be that patients included in this study were younger (mean of 48.2 years compared to 53.9 years) and thereby less likely to have developed CHD and increased risk of having a CAC score of zero. If more patients had a CAC score above zero, hypertension might have showed correlation with the CAC score as seen in previous studies [34]. The number of patients with a CAC score of zero was not reported in the study by Kugathasan et al., however the percentage prevalence of CAC scores in the range 0-99 (88.0\%) were comparable to the present study (89.0\%)Previous studies have shown both inverse correlations between BMI and CAC [37] and positive correlations between BMI and the 
Table 4 Linear regression on coronary artery calcium measured as log-transformed CAC scores above zero $(n=49)$

\begin{tabular}{|c|c|c|c|c|c|c|}
\hline \multirow[t]{2}{*}{ Variables } & \multicolumn{3}{|l|}{ Univariate } & \multicolumn{3}{|l|}{ Multivariate } \\
\hline & $\begin{array}{l}\text { Coeff } \\
(95 \% \mathrm{Cl})\end{array}$ & $\begin{array}{l}\text { Percent increase in } \\
\text { CAC score }(95 \% \mathrm{Cl})^{\mathrm{a}}\end{array}$ & $P$-value & $\begin{array}{l}\text { Coeff } \\
(95 \% \mathrm{Cl})\end{array}$ & Percent increase in CAC score $(95 \% \mathrm{CI})^{\mathrm{a}}$ & $P$-value \\
\hline Age & $0.06(0.03-0.09)$ & $15 \%(7-24 \%)$ & $<0.001$ & $0.07(0.04-0.10)$ & $16 \%(9-25 \%)$ & $<0.001$ \\
\hline Male sex & $-0.35(-0.91-0.22)$ & $-55 \%(-88-164 \%)$ & 0.222 & $-0.05(-0.53-0.44)$ & $-11 \%(68-173 \%)$ & 0.839 \\
\hline Dyslipidemia & $0.08(-0.60-0.76)$ & $19 \%(-75-472 \%)$ & 0.822 & $0.17(-0.40-0.73)$ & $47 \%(-60-436 \%)$ & 0.556 \\
\hline Diabetes & $0.76(0.20-1.33)$ & $577 \%(57-2020 \%)$ & 0.009 & $0.71(0.23-1.20)$ & $419 \%(68-1500 \%)$ & 0.005 \\
\hline Smoking & $0.05(-0.55-1.25)$ & $126 \%(-72-1695 \%)$ & 0.434 & $0.55(-0.19-1.28)$ & $250 \%(-35-1792 \%)$ & 0.140 \\
\hline
\end{tabular}

${ }^{a}$ Calculated as inversing log of the coefficients from the linear regression of log-transformed CAC scores and subtracting 1.

presence of CAC [38] suggesting a complex relationship between body size and the CAC score possibly explaining conflicting findings. Lastly, the mechanism of developing CAC could differ between patients with schizophrenia, bipolar disorder (included in Kugathasan et al.) and the general population explaining differences in correlation between the CAC score, BMI and hypertension. However, Kugathasan et al. found no differences in CAC scores between patients diagnosed with schizophrenia or bipolar disorder and the background population [20]. Thus, the findings of CAC scores in relation to norm percentiles in this study are consistent with the existing literature on $\mathrm{CAC}$ scores in patients with schizophrenia.

We found a high prevalence $(69.9 \%)$ of zero CAC score which has shown to be associated with lower allcause mortality, lower rate of cardiovascular events and improved survival compared to a CAC score above zero $[26,39,40]$. In younger asymptomatic individuals aged $\leq 45$ years, an adjusted all-cause mortality hazard ratio (HR) of 2.3 has been reported for individuals with even mild coronary artery calcium (CAC score 1-100) compared to individuals with no coronary artery calcium [41]. Hence, having CAC score of zero is interpreted as low risk of $\mathrm{CHD}$ and all-cause mortality in a clinical setting, which would be the case in $69.9 \%$ of the patients with schizophrenia included in this study. In contrast, several studies support higher prevalence of CHD and related mortality in patients with schizophrenia compared to the general population $[1,7,42]$. One explanation for the findings could be the high prevalence of smoking found in the present study (76.1\%) given higher mortality rates in smokers compared to nonsmokers in the general population even in the absence of coronary artery calcium and underestimation of CHD risk in smokers [43, 44]. The underestimation in smokers challenges the use of CAC score for estimating cardiovascular risk in patients with schizophrenia due to the increased prevalence of smoking [6]. Moreover, increased mortality rates were found in patients with schizophrenia or bipolar disorder irrespective of the CAC score with high rates of non-cardiovascular causes of death [20] suggesting minor importance of the CAC score in this population. The high prevalence of dyslipidemia (66.9\%) found in this study further suggests competing risks of CHD with no association to the CAC score. Furthermore, an increased likelihood of not showing up to appointments at general practitioner and negative symptoms as part of the psychopathology such as social deprivation in patients with schizophrenia [45] could cause less control of blood lipids and less use of lipid-lowering treatment [11].

Another possible explanation for the high number of patients having a CAC score of zero could be a more frequent development of unstable CHD in patients with schizophrenia. Unstable CHD has shown to be primarily related to vulnerable atherosclerotic plaques that cannot be detected by CCT instead of dense calcified plaques

Table 5 Sensitivity analysis on CAC scores in multivariate logistic regression ( $N=163)$ and log-transformed CAC scores above zero in linear $(n=49)$ regression

\begin{tabular}{|c|c|c|c|c|}
\hline \multirow[t]{2}{*}{ Variables } & \multicolumn{2}{|c|}{ Logistic regression } & \multicolumn{2}{|l|}{ Linear regression } \\
\hline & OR $(95 \% \mathrm{Cl})$ & $P$-value & Coeff & $P$-value \\
\hline$\overline{\mathrm{LDL}}$ & $1.21(0.69-2.11)$ & 0.505 & $-1.93(-0.48-0.09)$ & 0.178 \\
\hline Triglyceride & $1.05(0.87-1.27)$ & 0.609 & $-0.01(-0.08-0.07)$ & 0.849 \\
\hline $\mathrm{HDL}$ & $0.93(0.31-2.83)$ & 0.905 & $-0.07(-0.60-0.46)$ & 0.805 \\
\hline Statin treatment & $1.38(0.47-4.05)$ & 0.561 & $-0.08(-0.58-0.41)$ & 0.733 \\
\hline $\mathrm{HbA1c}$ & $1.01(0.97-1.05)$ & 0.770 & $0.02(-0.00-0.04)$ & 0.101 \\
\hline Antidiabetic medication & $1.69(0.55-5.17)$ & 0.357 & $0.66(0.15-1.18)$ & 0.013 \\
\hline
\end{tabular}


[46]. The stabilizing role of coronary artery calcium in the atherosclerotic plaques might not be developed to the same extent in patients with schizophrenia due to a more rapid development of atherosclerosis. Moreover, the overall low amount of coronary calcium found in this study could possibly be explained by the relatively low mean age of the population (48.2 years) and patients as young as 24 years less likely to have developed detectable and calcified plaques. On the other hand, statin treatment has shown to facilitate plaque stabilization by progression of coronary artery calcium and thereby decrease the risk of future cardiovascular events [47], which could confound the results of this study in patients with high CAC scores since treatment with lipid lowering medication was included in the definition of dyslipidemia.

\section{Strengths and limitations}

As the first study ever investigating the CAC score in a sample of patients with schizophrenia, who were not referred for CCT, thus eliminating referral bias, this study has several strengths. Data collection was conducted in a structured manner enabling definitions of diabetes, dyslipidemia and risk assessment according to gold standards. In addition, the population was characterized thoroughly, reporting the duration of schizophrenia, contact with outpatient clinics, treatment with clozapine and severity at the time of examination according to a standardized rating scale.

However, the findings of this study should be interpreted in the light of its limitations. Especially, results from the linear regression should be interpreted with caution due to the large amount of CAC scores of zero and thereby a small sample size and broad $95 \%$ CIs. The current data did not allow for further statistical test of the CAC score between the general population and patients with schizophrenia due to the distributional properties of the CAC score. Furthermore, age- and gender matched norm percentiles used in this study were based on an American population from Raggi et al. [25] described in studies by Valenti et al. [26] and Ronde et al [24]. and, therefore, the generalizability to Denmark might be limited. However, these percentiles were used in Danish clinical practice, and a recent meta-analysis pooling several CAC score nomograms suggested that median CAC scores for USA and Europe were similar [24]. Patients with known cardiovascular disease or symptoms were not excluded from this study and might not be comparable with the norm reference population. Data on smoking and medication was self-reported, i.e. response bias cannot be ruled out. Moreover, the data on smoking was limited, categorizing patients as previous or current smokers with no consideration of the amount or duration. In addition, selection bias may have occurred in terms of not including the most severe cases of schizophrenia due to inability to attend a long and demanding examination program. Patients with the most severe schizophrenia might have the poorest lifestyle and the highest CAC score and are possibly not included in this study. However, a mean duration of schizophrenia of 19.7 years with patients still having contact with outpatient clinics despite the long duration indicates severe disease. Results from the CGI-SCH rating scale showed an overall high severity of disease in the study population with a total of $86(55 \%)$ patients categorized as either moderately, markedly, severely or among the most extremely ill. In addition, 41 (25.2\%) patients received clozapine treatment indicating severe disease in terms of resistance to treatment and possibly higher severity than classified by the CGI-SCH rating scale. Thus, selection bias does not fully explain the finding of a high number of patients with a CAC score of zero, and it will be challenging to include patients with even more severe disease in future studies. Furthermore, patients with the highest CAC score might have died and therefore not been included in this study.

The Agatston scoring method used for CAC scoring in this study has been discussed in the literature, and some limitations have been recognized [30]. The total number of calcified plaques, the presence of coronary artery calcium in the proximal dominant coronary artery and increased number of coronary arteries with calcium have shown to be predictive of CHD-related events independently of the CAC score [48-50]. However, the Agatston scoring method is considered the gold standard for detection of coronary artery calcium [30].

In conclusion, the findings of this study support previous findings that predictors of $\mathrm{CHD}$ used in the general population might not be sufficient in patients with schizophrenia $[19,51]$. Future studies should explore other measures of subclinical CHD to improve and initiate early prophylactic treatment and may include measures of peripheral atherosclerosis or cardiac autonomic neuropathy to improve early detection and intervention.

\section{Conclusions}

Coronary artery calcium in patients with schizophrenia follows norm percentiles compared to the general population. Variables associated with the presence and extent of coronary artery calcium in the general population are similarly associated with coronary artery calcium in patients with schizophrenia. Further studies should address the progression of coronary artery calcium and other measures of subclinical cardiovascular disease in these patients, e.g. peripheral atherosclerosis or cardiac autonomic neuropathy to improve treatment and survival. 


\section{Abbreviations}

CHD: Coronary heart disease; CAC: Coronary artery calcium; CCT: Cardiac computed tomography; ICD-10: International Classification of Diseases, Tenth Revision; CGI-SCH: Clinical Global Impression-Schizophrenia scale; HbA1c: Glycated hemoglobin; HDL: High-density lipoprotein; LDL: Lowdensity lipoprotein

\section{Supplementary Information}

The online version contains supplementary material available at https://doi. org/10.1186/s12888-021-03412-x.

Additional file 1: Supplementary Fig. 1. This chart is for use in people without cardiovascular disease, diabetes or very high levels of individual risk factors [1]. Supplementary Fig. 2. This chart is for use in younger people under the age of 40 since the absolute risk chart may underestimate cardiovascular risk in this population [2].

\section{Acknowledgements}

Not applicable.

\section{Authors' contributions}

TT collected data and evaluated the cardiac CT scans, analyzed and interpreted the data and was a major contributor in writing the manuscript, including writing the initial draft and implementing suggestions from the co-authors. RA revised the manuscript and contributed to the visualization of the data. SEJ contributed with conceptualization, project administration, investigation, collection and visualization of data, funding acquisition, supervision, development of the study design including aims, analyzed the cardiac CT scans and was a major contributor to writing the manuscript. SG revised the manuscript and contributed with data curation, statistical analysis, visualization and interpretation of data. JBF made it possible to carry out the cardiac CT scans and provided analysis tools for determining the coronary artery calcium scores, contributed with the technical details of the scanner and revised the manuscript. CP contributed to the collection, interpretation and visualization of the data and revised the manuscript. REN contributed with conceptualization, project administration, investigation, collection and visualization of data, funding acquisition, supervision, development of the study design including aims and was a major contributor to writing the manuscript. All authors read and approved the final manuscript.

\section{Funding}

The collection, analysis and interpretation of data presented in this study were supported by the Danish Heart Foundation (grant number 15-R99A5870-22926), Brødrene Hartmann Foundation (grant number A72332), Arvid Nielssons Foundation (grant number 1073808), the Obel Family Foundation (grant numbers 27744, 30123), Aalborg University Hospital, Psychiatric Hospital, Denmark (grant number 2011-125244) and the Lundbeck Foundation (TT, grant number 2019-017596).

\section{Availability of data and materials}

The datasets generated and analyzed during the current study are not publicly available but are available from the corresponding author upon reasonable request.

\section{Declarations}

Ethics approval and consent to participate

The study was approved by the Regional Committee on Biomedical Research Ethics of North Jutland (N-20140047). All participants and legal caretakers gave informed consent, and the study was conducted according to the declaration of Helsinki. The categories of the person data collected for the project are registered in the processing activities of research in the North Denmark Region in compliance with EU GDPR article 30. The study was registered at clinicaltrials.gov with identifier NCT02885792.

\section{Consent for publication}

Not applicable.

\section{Competing interests}

$\Pi T$ and RA declare that they have no competing interests. SEJ has received an unrestricted research grant from the Obel Family Foundation. SG, JBF and $\mathrm{CP}$ declare that they have no competing interests. REN has received research grants from $\mathrm{H}$. Lundbeck and Otsuka Pharmaceuticals for clinical trials, received speaking fees from Bristol-Myers Squibb, Astra Zeneca, Janssen \& Cilag, Lundbeck, Servier, Otsuka Pharmaceuticals, Teva A/S and Eli Lilly and has acted as advisor to Astra Zeneca, Eli Lilly, Lundbeck, Otsuka Pharmaceuticals, Takeda and Medivir, and has acted as investigator for Janssen-Cilag, Lundbeck, Boehringer, Compass and Sage.

\section{Author details}

${ }^{1}$ Department of Psychiatry, Aalborg University Hospital, Aalborg, Denmark. ${ }^{2}$ Department of Cardiology, Aalborg University Hospital, Aalborg, Denmark. ${ }^{3}$ Department of Clinical Medicine, Aalborg University, Aalborg, Denmark. ${ }^{4}$ Department of Radiology, Aalborg University Hospital, Aalborg, Denmark.

Received: 1 March 2021 Accepted: 3 August 2021

Published online: 23 August 2021

\section{References}

1. Plana-Ripoll O, Pedersen CB, Agerbo $E$, et al. A comprehensive analysis of mortality-related health metrics associated with mental disorders: a nationwide, register-based cohort study. Lancet. 2019;394:1827-35. https:// doi.org/10.1016/S0140-6736(19)32316-5.

2. Laursen TM, Plana-Ripoll O, Andersen PK, et al. Cause-specific life years lost among persons diagnosed with schizophrenia: is it getting better or worse? Schizophr Res. 2019;206:284-90. https://doi.org/10.1001/jamapsychiatry.2 014.2502

3. Nordentoft M, Wahlbeck K, Hällgren J, et al. Excess mortality, causes of death and life expectancy in 270, 770 patients with recent onset of mental disorders. PLoS One. 2013;8. https://doi.org/10.1371/journal.pone.0055176 Epub ahead of print

4. Pérez-Piñar M, Mathur R, Foguet $Q$, et al. Cardiovascular risk factors among patients with schizophrenia, bipolar, depressive, anxiety, and personality disorders. Eur Psychiatry. 2016;35:8-15. https://doi.org/10.1016/j.eurpsy.2016. 02.004 .

5. Correll CU, Solmi M, Veronese N, et al. Prevalence, incidence and mortality from cardiovascular disease in patients with pooled and specific severe mental illness: a large-scale meta-analysis of 3,211,768 patients and 113,383,368 controls. World Psychiatry. 2017;16:163-80. https://doi.org/10.1 002/wps.20420.

6. Vancampfort D, Stubbs B, Mitchell AJ, et al. Risk of metabolic syndrome and its components in people with schizophrenia and related psychotic disorders, bipolar disorder and major depressive disorder: a systematic review and meta-analysis. World Psychiatry. 2015;14:339-47. https://doi. org/10.1002/wps.20252.

7. Westman J, Eriksson SV, Gissler M, et al. Increased cardiovascular mortality in people with schizophrenia: a 24-year national register study; 2019. p. 51927. https://doi.org/10.1017/S2045796017000166.

8. Speyer H, Christian Brix Nørgaard H, Birk M, et al. The CHANGE trial: no superiority of lifestyle coaching plus care coordination plus treatment as usual compared to treatment as usual alone in reducing risk of cardiovascular disease in adults with schizophrenia spectrum disorders and abdominal obesity. World Psychiatry. 2016;15:155-65. https://doi.org/10.1 002/wps.20318.

9. Amiri M, Janssen F, Kunst AE. The decline in ischaemic heart disease mortality in seven European countries: exploration of future trends. J Epidemiol Community Health. 2011:676-81. https://doi.org/10.1136/jech.201 0.109058 .

10. Mitchell AJ, Lawrence D. Revascularisation and mortality rates following acute coronary syndromes in people with severe mental illness: comparative meta-analysis. Br J Psychiatry. 2011;198:434-41. https://doi. org/10.1192/bjp.bp.109.076950.

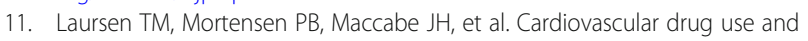
mortality in patients with schizophrenia or bipolar disorder: a Danish population-based study. Psychol Med. 2014;44:1625-37. https://doi.org/10.1 017/S003329171300216X.

12. Nielsen J, Juel J, Ks A, et al. Unrecognised myocardial infarction in patients with schizophrenia †. 2019. https://doi.org/10.1017/neu.2014.41 Epub ahead of print. 
13. Rosenthal SH, Porter KA, Coffey B. Pain insensitivity in schizophrenia. Case report and review of the literature. Gen Hosp Psychiatry. 1990;12:319-22. https://doi.org/10.1016/0163-8343(90)90050-M.

14. Joshi PH, Patel B, Blaha MJ, et al. Coronary artery calcium predicts cardiovascular events in participants with a low lifetime risk of cardiovascular disease: the multi-ethnic study of atherosclerosis (MESA). Atherosclerosis. 2016;246:367-73. https://doi.org/10.1016/j.atherosclerosis.2 016.01.017.

15. Nasir K, Rubin J, Blaha MJ, et al. Interplay of coronary artery calcification and traditional risk factors for the prediction of all-cause mortality in asymptomatic individuals. Circ Cardiovasc Imaging. 2012;5:467-73. https:// doi.org/10.1161/CIRCIMAGING.111.964528.

16. Shaw LJ, Giambrone AE, Blaha MJ, et al. Long-term prognosis after coronary artery calcification testing in asymptomatic patients: a cohort study. Ann Intern Med. 2015;163:14-21. https://doi.org/10.7326/M14-0612.

17. Gheorghe AG, Jacobsen C, Thomsen R, et al. Coronary artery CT calcium score assessed by direct calcium quantification using atomic absorption spectroscopy and compared to macroscopic and histological assessments. Int J Legal Med. 2019;133:1485-96. https://doi.org/10.1007/s00414-018-01 998-8.

18. Alashi A, Lang $R$, Seballos $R$, et al. Reclassification of coronary heart disease risk in a primary prevention setting: traditional risk factor assessment vs. coronary artery calcium scoring. Cardiovasc Diagn Ther. 2019;9:214-20. https://doi.org/10.21037/cdt.2019.04.05.

19. Osborn DPJ, Hardoon S, Omar RZ, et al. Cardiovascular risk prediction models for people with severe mental illness results from the prediction and management of cardiovascular risk in people with severe mental illnesses (PRIMROSE) research program. JAMA Psychiatry. 2015;72:143-51. https://doi.org/10.1001/jamapsychiatry.2014.2133.

20. Kugathasan $P$, Johansen $M B$, Jensen $M B$, et al. Coronary artery calcification and mortality risk in patients with severe mental illness. Circ Cardiovasc Imaging. 2019;12:1-8. https://doi.org/10.1161/CIRCIMA GING.118.008236.

21. Pletcher MJ, Tice JA, Pignone M, et al. What does my patient's coronary artery calcium score mean? Combining information from the coronary artery calcium score with information from conventional risk factors to estimate coronary heart disease risk. BMC Med. 2004;2:31. https://doi.org/1 $0.1186 / 1741-7015-2-31$

22. Hecht HS, Blaha MJ, Kazerooni EA, et al. CAC-DRS: coronary artery calcium data and reporting system. An expert consensus document of the Society of Cardiovascular Computed Tomography (SCCT). J Cardiovasc Comput Tomogr. 2018;12:185-91. https://doi.org/10.1016/j.jcct.2018.03.008.

23. Piepoli MF, Hoes AW, Agewall S, et al. 2016 European guidelines on cardiovascular disease prevention in clinical practice. Eur Heart J. 2016;37: 2322-30. https://doi.org/10.1093/eurheartj/ehw106 2334-2335.

24. de Ronde MWJ, Khoshiwal A, Planken RN, et al. A pooled-analysis of age and sex based coronary artery calcium scores percentiles. J Cardiovasc Comput Tomogr. 2020;14:414-20. https://doi.org/10.1016/j.jcct.2020.01.006

25. Raggi $\mathrm{P}$, Callister TQ, Cooil B, et al. Clinical investigation and reports identification of patients at increased risk of first unheralded acute myocardial infarction by electron-beam computed tomography. 2000:850-5. https://doi.org/10.1161/01.cir.101.8.850.

26. Valenti V, Ó Hartaigh B, Heo R, et al. A 15-year warranty period for asymptomatic individuals without coronary artery calcium: a prospective follow-up of 9715 individuals. JACC Cardiovasc Imaging. 2015:8:900-9. https://doi.org/10.1016/j.jcmg.2015.01.025.

27. McClelland RL, Chung H, Detrano R, et al. Distribution of coronary artery calcium by race, gender, and age: results from the multi-ethnic study of atherosclerosis (MESA). Circulation. 2006;113:30-7. https://doi.org/10.1161/ CIRCULATIONAHA.105.580696.

28. Aagaard J, Kugathasan P, Jensen SE. Coronary artery disease as a cause of morbidity and mortality in patients suffering from schizophrenia: protocol for a prospective cohort study with long-term follow-up. Wolters Kluwer MEdknow. 2016. https://doi.org/10.4103/2468-5658.196979 Epub ahead of print.

29. Agatston AS, Janowitz FWR, Hildner FJ, et al. Quantification of coronary artery calcium using ultrafast computed tomography. J Am Coll Cardiol. 1990;15:827-32. https://doi.org/10.1016/0735-1097(90)90282-T.

30. Blaha MJ, Mortensen MB, Kianoush S, et al. Coronary artery calcium scoring: is it time for a change in methodology? JACC Cardiovasc Imaging. 2017;10: 923-37. https://doi.org/10.1016/j.jcmg.2017.05.007.
31. Haro JM, Kamath SA, Ochoa S, et al. The clinical global impressionschizophrenia scale: a simple instrument to measure the diversity of symptoms present in schizophrenia. Acta Psychiatr Scand Suppl. 2003;107: 16-23. https://doi.org/10.1034/j.1600-0447.107.s416.5.x.

32. Mach F, Baigent C, Catapano AL, et al. 2019 ESC/EAS guidelines for the management of dyslipidaemias: lipid modification to reduce cardiovascular risk. Eur Heart J. 2019;48:121-7. https://doi.org/10.1093/ eurheartj/ehz455.

33. Gudmondsson EF, Gudnason V, Launer LJ, et al. Coronary artery calcium distributions in older persons in the AGES-Reykjavik study. Eur J Epidemiol. 2012;27:673-87. https://doi.org/10.1007/s10654-012-9730-6.

34. Grossman C, Shemesh J, Dovrish Z, et al. Coronary artery calcification is associated with the development of hypertension. Am J Hypertens. 2013;26: 13-9. https://doi.org/10.1093/ajh/hps028.

35. Weinberg RL, Rubenfire M, Brook RD. Coronary artery calcium scoring in patients with hypertension. J Hum Hypertens. 2020;34:609-16. https://doi. org/10.1038/s41371-020-0350-4

36. Cp Diederichsen A, Sand NP, Nørgaard B, et al. Discrepancy between coronary artery calcium score and HeartScore in middle-aged Danes: the DanRisk study. Eur J Prev Cardiol. 2012;19:558-64. https://doi.org/10.1177/1 741826711409172.

37. Kovacic JC, Lee $P$, Baber $U$, et al. Inverse relationship between body mass index and coronary artery calcification in patients with clinically significant coronary lesions. Atherosclerosis. 2012;221:176-82. https://doi.org/10.1016/j.a therosclerosis.2011.11.020.

38. Aljizeeri A, Coutinho T, Pen A, et al. Obesity and coronary artery calcification: can it explain the obesity-paradox? Int J Card Imaging. 2015;31:1063-70. https://doi.org/10.1007/s10554-015-0643-9.

39. Blaha M, Budoff MJ, Shaw $L$, et al. Absence of coronary artery calcification and all-cause mortality. JACC Cardiovasc Imaging. 2009;2:692-700. https:// doi.org/10.1016/j.jcmg.2009.03.009.

40. Sarwar A, Shaw LJ, Shapiro MD, et al. Diagnostic and prognostic value of absence of coronary artery calcification. JACC Cardiovasc Imaging. 2009;2: 675-88. https://doi.org/10.1016/j.jcmg.2008.12.031.

41. Tota-Maharaj R, Blaha MJ, McEvoy JW, et al. Coronary artery calcium for the prediction of mortality in young adults $<45$ years old and elderly adults $>$ 75 years old. Eur Heart J. 2012;33:2955-62. https://doi.org/10.1093/eurheartj/ ehs 230 .

42. Laursen TM, Munk-Olsen T, Vestergaard M. Life expectancy and cardiovascular mortality in persons with schizophrenia. Curr Opin Psychiatry. 2012;25:83-8. https://doi.org/10.1097/YCO.0b013e3283503 $5 \mathrm{ca}$.

43. McEvoy JW, Blaha MJ, Rivera JJ, et al. Mortality rates in smokers and nonsmokers in the presence or absence of coronary artery calcification. JACC Cardiovasc Imaging. 2012;5:1037-45. https://doi.org/10.1016/j.jcmg.2 012.02.017.

44. Zimmermann GS, Rüther $T$, Ziegler $F$, et al. Predictive value of coronary calcifications for future cardiac events in asymptomatic patients: underestimation of risk in asymptomatic smokers. Int J Card Imaging. 2019; 35:1387-93. https://doi.org/10.1007/s10554-019-01571-3.

45. Nørgaard HCB, Schou Pedersen H, Fenger-Grøn M, et al. Schizophrenia and attendance in primary healthcare: a population-based matched cohort study. Scand J Prim Health Care. 2019;37:358-65. https://doi.org/10.1080/02 813432.2019.1639927.

46. Leber AW, Knez A, White CW, et al. Composition of coronary atherosclerotic plaques in patients with acute myocardial infarction and stable angina pectoris determined by contrast-enhanced multislice computed tomography. Am J Cardiol. 2003;91:714-8. https://doi.org/10.1016/S0002-914 9(02)03411-2.

47. Henein M, Granåsen G, Wiklund U, et al. High dose and long-term statin therapy accelerate coronary artery calcification. Int J Cardiol. 2015. https://doi.org/10.1016/j.ijcard.2015.02.072 Epub ahead of print.

48. Berry A, Drake RJ, Webb RT, et al. Investigating the agreement between cardiovascular disease risk calculators among people diagnosed with schizophrenia. Front Psychiatry. 2018:9:1-9. https://doi.org/10.3389/fpsyt.201 8.00685 .

49. Arnson $\mathrm{Y}$, Rozanski A, Gransar $\mathrm{H}$, et al. Comparison of the coronary artery calcium score and number of calcified coronary plaques for predicting patient mortality risk. Am J Cardiol. 2017;120:2154-9. https://doi.org/10.101 6/j.amjcard.2017.09.001. 
50. Blaha MJ, Budoff MJ, Tota-maharaj R, et al. Improving the CAC score by addition of regional measures of calcium distribution; 2016. p. 9. https://doi. org/10.1016/j.jcmg.2016.03.001. Epub ahead of print

51. Ferencik M, Pencina KM, Liu T, et al. Coronary artery calcium

distribution is an independent predictor of incident major coronary heart disease events: results from the Framingham heart study. Circ

Cardiovasc Imaging. 2017;10:1-21. https://doi.org/10.1161/CIRCIMA GING.117.006592.

\section{Publisher's Note}

Springer Nature remains neutral with regard to jurisdictional claims in published maps and institutional affiliations.

Ready to submit your research? Choose BMC and benefit from:

- fast, convenient online submission

- thorough peer review by experienced researchers in your field

- rapid publication on acceptance

- support for research data, including large and complex data types

- gold Open Access which fosters wider collaboration and increased citations

- maximum visibility for your research: over $100 \mathrm{M}$ website views per year

At $B M C$, research is always in progress.

Learn more biomedcentral.com/submissions 